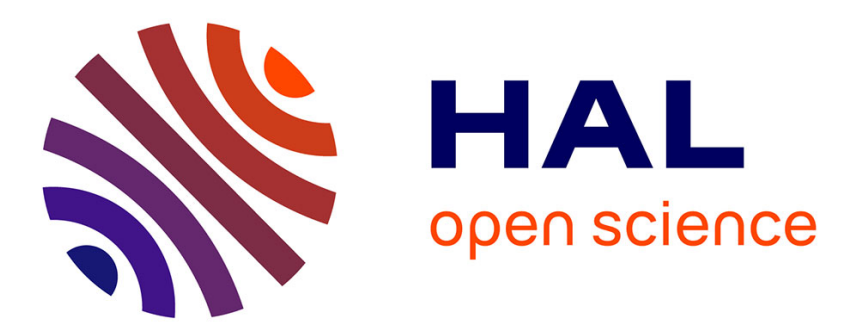

\title{
Superadiabatic evolution and adiabatic transition probability between two nondegenerate levels isolated in the spectrum
}

Alain Joye, Charles-Edouard Pfister

\section{- To cite this version:}

Alain Joye, Charles-Edouard Pfister. Superadiabatic evolution and adiabatic transition probability between two nondegenerate levels isolated in the spectrum. Journal of Mathematical Physics, 1993, 34 (2), 10.1063/1.530255 . hal-01221129

\section{HAL Id: hal-01221129 \\ https://hal.science/hal-01221129}

Submitted on 27 Oct 2015

HAL is a multi-disciplinary open access archive for the deposit and dissemination of scientific research documents, whether they are published or not. The documents may come from teaching and research institutions in France or abroad, or from public or private research centers.
L'archive ouverte pluridisciplinaire HAL, est destinée au dépôt et à la diffusion de documents scientifiques de niveau recherche, publiés ou non, émanant des établissements d'enseignement et de recherche français ou étrangers, des laboratoires publics ou privés. 


\title{
Superadiabatic evolution and adiabatic transition probability between two nondegenerate levels isolated in the spectrum
}

\author{
Alain Joye \\ Département de Physique, Ecole Polytechnique Fédérale de Lausanne, \\ CH-1015 Lausanne, Switzerland \\ Charles-Edouard Pfister \\ Département de Mathématiques, Ecole Polytechnique Fédérale de Lausanne, \\ CH-1015 Lausanne, Switzerland
}

(Received 26 June 1992; accepted for publication 16 September 1992)

\begin{abstract}
The Schrödinger equation in the adiabatic limit when the Hamiltonian depends analytically on time and possesses for any fixed time two nondegenerate eigenvalues $e_{1}(t)$ and $e_{2}(t)$ bounded away from the rest of the spectrum is considered herein. An approximation of the evolution called superadiabatic evolution is constructed and studied. Then a solution of the equation which is asymptotically an eigenfunction of energy $e_{1}(t)$ when $t \rightarrow-\infty$ is considered. Using superadiabatic evolution, an explicit formula for the transition probability to the eigenstate of energy $e_{2}(t)$ when $t \rightarrow+\infty$, provided the two eigenvalues are sufficiently isolated in the spectrum, is derived. The end result is a decreasing exponential in the adiabaticity parameter times a geometrical prefactor.
\end{abstract}

\section{INTRODUCTION}

During the last years several theoretical and experimental works have been devoted to different aspects of the adiabatic regime of quantum dynamics. We consider here two aspects of this physically important regime: the construction of approximate evolutions of the Schrödinger equation called "superadiabatic evolutions" and the explicit computation of the probability of a transition between two nondegenerate energy levels, isolated in the spectrum of the Hamiltonian. From the mathematical point of view the adiabatic limit for time-dependent systems is of the same kind as the semiclassical limit for stationary systems. It is a singular limit which cannot be tackled by usual perturbation methods. Recently a mathematical theory of the two considered aspects has been developed when the time-dependent Hamiltonian is analytic in time. The transition probability for two-level systems has been studied in Refs. 1-3 and was shown to be exponentially small in the adiabaticity parameter for general systems in Ref. 4. On the other hand, the existence of superadiabatic evolutions has been shown in Ref. 5 . However, no general formula for the transition probability between isolated levels in the adiabatic limit can be found in the literature. It is the goal of this paper to establish such a formula for general (unbounded) Hamiltonians.

The paper is divided into two parts. We first construct a superadiabatic evolution which approximates the true evolution of the Schrödinger equation up to exponentially small correction terms with respect to the parameter controlling the adiabatic regime. Once the superadiabatic evolution is defined, we show that the computation of the transition probability of the initial problem reduces to an effective two-dimensional problem, which we analyze as in Ref. 1 .

The concept of superadiabatic evolution can be traced back up to a paper of Garrido, ${ }^{6}$ who considered evolutions different from the usual adiabatic evolution which he used to obtain higher-order corrections to the adiabatic theorem of quantum mechanics. But it is Berry ${ }^{7,8}$ who emphasized the importance of such evolutions in the present problem and introduced the 
concept of superadiabatic evolutions. Independently of Berry's work, Nenciu ${ }^{5}$ introduced similar ideas, in a more general setting and controlled the correction terms, showing in this way the existence of these evolutions. We construct our superadiabatic evolution iteratively, as in Refs. $6-8$, but using the simple iteration scheme of Ref. 2 . The iterative schemes of Refs. 6-8 or 5 are equivalent to ours which is however more suitable for the kind of estimates we are doing. In particular, the dependence of our estimates with respect to the spectrum of the Hamiltonian is better than in Ref. 5. This improvement is important for the second part of the paper, in which we compute the transition probability between two nondegenerate energy levels by means of our superadiabatic evolution. The computation is done for the following scattering experiment: the system is prepared at time $t=-\infty$ in an eigenstate of one of the energy levels and the transition is measured at time $t=\infty$. The transition probability is known to be exponentially small in this context, ${ }^{4}$ but here our result is an explicit formula for this transition probability, which has the same structure as our formula for two-level systems in Ref. 1: it is the product of an exponentially small term in $\epsilon$, which is expressed through the analytic continuation of the energy levels into the complex-time plane, with a factor independent of $\epsilon$, which has a geometrical origin similar to the Berry phase. ${ }^{9,1,10}$ The exponential decay rate is the same as for two-level systems but in the actual computation of the geometrical prefactor we must now take into account the global "rotation" in the Hilbert space of the spectral subspace spanned by the two eigenvectors associated with $e_{1}(t)$ and $e_{2}(t)$.

\section{SUPERADIABATIC EVOLUTION}

Let $\mathscr{H}$ be a separable complex Hilbert space, and $H(t), t \in \mathbb{R}$, a family of self-adjoint operators, bounded from below, defined on a common dense domain $D \subset \mathscr{H}$. The timedependent Schrödinger equation in the adiabatic regime can be written as

$$
i \epsilon \frac{\partial}{\partial t} \varphi_{\epsilon}(t)=H(t) \varphi_{\epsilon}(t), \quad \varphi_{\epsilon}(s)=\varphi^{*} \in D
$$

Here $t$ is a rescaled time and $\epsilon$ a small positive parameter, which controls the adiabatic regime. The adiabatic limit corresponds to the singular limit $\epsilon \rightarrow 0$. We study this equation under the following two hypotheses:

(I) Analyticity: There is an open neighborhood $\Omega=\{t+i s:|s|<r\}$ of the real axis, such that for each $z \in \Omega, H(z)$ is a closed operator defined on $D$, and for each $\varphi \in D$ the function $H(z) \varphi$ is analytic in $z \in \Omega$.

(II) Separation of the spectrum: For each $t \in \mathbb{R}$ the spectrum $\sigma(t)$ of $H(t)$ is divided into two parts $\sigma_{1}(t)$ and $\sigma_{2}(t), \sigma_{1}(t)$ being bounded, so that the sets in $\mathbb{R}^{2}$

$$
\mathscr{B}_{i}:=\left\{(t, \lambda): \lambda \in \sigma_{i}(t), t \in \mathbb{R}\right\}, \quad i=1,2
$$

are disjoint. The distance between $\sigma_{1}(t)$ and $\sigma_{2}(t)$ is larger than some positive constant $g$.

The spectral projector associated with the spectral subset $\sigma_{1}(t)$ is denoted by $Q(t)$. The solution $\varphi(t)$ of the Schrödinger equation can be expressed as

$$
\varphi(t)=U(t, s) \varphi^{*}
$$

where $U(t, s)$ is a two-parameter family of unitary operators, strongly continuous in $t$ and $s$ and which leave the domain $D$ invariant. For all $t_{1}, t_{2}, t_{3}$

$$
U\left(t_{1}, t_{2}\right) U\left(t_{2}, t_{3}\right)=U\left(t_{1}, t_{3}\right), \quad U\left(t_{1}, t_{1}\right)=\mathbb{I}
$$

and $U$ is strongly differentiable in $t$ and $s$ on the domain $D$, 


$$
i \epsilon \frac{\partial}{\partial t} U(t, s)=H(t) U(t, s)
$$

and

$$
i \epsilon \frac{\partial}{\partial s} U(t, s)=-U(t, s) H(s)
$$

We call $U$ the evolution associated with the Eq. (2.1) (see, e.g., Ref. 11, Chap. 2).

Let $P(t)$, be a smooth family of projectors, which decompose the Hilbert space

$$
\mathscr{H}=P(t) \mathscr{H} \oplus(\mathbb{I}-P(t)) \mathscr{H} .
$$

We say that an evolution $V$ is compatible with the decomposition of the Hilbert space if for all $t$ and $s$

$$
P(t) V(t, s)=V(t, s) P(s) .
$$

Under the assumption II the adiabatic evolution is compatible with the decomposition of the Hilbert space given by the spectral projectors $Q(t)$ and $\mathbb{I}-Q(t)$. It is defined as the solution of the equation

$$
i \epsilon \frac{\partial}{\partial t} V(t, s)=\left(H(t)+i \epsilon\left[Q^{\prime}(t), Q(t)\right]\right) V(t, s), \quad V(s, s)=\mathbb{I},
$$

where ' denotes the derivative with respect to $t$. It approximates the true evolution $U$ up to correction terms of order $O(\epsilon)$ : for all $t$ and $s$ there exists a constant $M$ such that

$$
\|U(t, s)-V(t, s)\|<M|t-s| \epsilon .
$$

Since the adiabatic evolution is compatible with the decomposition of the Hilbert space given by the spectral projections $Q(t)$ and $\mathbb{I}-Q(t)$, a transition from one of these spectral subspaces into the other one is of order $O\left(\epsilon^{2}\right)$. The main purpose of this section is to construct another smooth decomposition of $\mathscr{H}$ into

$$
\mathscr{H}=Q_{*}(t) \mathscr{H} \oplus\left(\mathbb{I}-Q_{*}(t)\right) \mathscr{H}
$$

and another evolution $V_{*}(t, s)$ compatible with this decomposition,

$$
Q_{*}(t) V_{*}(t, s)=V_{*}(t, s) Q_{*}(s)
$$

and such that for all $t$ and $s$ there exist a constant $M<\infty$ and a constant $\tau>0$ with the property

$$
\left\|U(t, s)-V_{*}(t, s)\right\|<M|t-s| \exp \left(-\epsilon^{-1} \tau\right) .
$$

Such an evolution is called a superadiabatic evolution. The projector $Q_{*}(t)$ is a spectral projector of a Hamiltonian $H_{*}(t)$ which we define by iteration in the next section, starting with $H(t)$. The evolution $V_{*}$ is the solution of

$$
i \epsilon \frac{\partial}{\partial t} V_{*}(t, s)=\left(H_{*}(t)+i \epsilon\left[Q_{*}^{\prime}(t), Q_{*}(t)\right]\right) V_{*}(t, s), \quad V_{*}(s, s)=\mathbb{I}
$$

Note that both $V_{*}$ and the projector $Q_{*}$ are $\epsilon$ dependent. 


\section{A. Iteration scheme}

We study here the iteration scheme, which gives the operator $H_{*}(t)$. The construction is local, and we consider it in an open fixed disc $D(z, \eta)=\left\{z^{\prime} \in \mathbb{C}|| z^{\prime}-z \mid<\eta\right\} \subset \Omega$. We make the hypothesis

(III) There exists a simple closed path $\Gamma$ in the complex plane, counterclockwise oriented, such that for all $z^{\prime} \in D(z, \eta)$ the spectrum $\sigma\left(z^{\prime}\right)$ of $H\left(z^{\prime}\right)$ can be divided into two disjoint parts $\sigma_{1}\left(z^{\prime}\right)$ and $\sigma_{2}\left(z^{\prime}\right)$, with $\sigma_{1}\left(z^{\prime}\right)$ in the interior of $\Gamma$.

If $R(z, \lambda)=(H(z)-\lambda)^{-1}$, then we can write for all $z^{\prime} \in D(z, \eta)$ the spectral projector $Q\left(z^{\prime}\right)$ associated with $\sigma_{1}\left(z^{\prime}\right)$ as

$$
Q\left(z^{\prime}\right)=-\frac{1}{2 \pi i} \oint_{\Gamma} R\left(z^{\prime}, \lambda\right) d \lambda
$$

We now motivate the iteration scheme of Ref. 2. Let us consider the true evolution $U(t, s)$. The projector $Q(s)$ evolves to the projector $Q_{\epsilon}(t)=U(t, s) Q(s) U(s, t)$ at time $t$, and $Q_{\epsilon}(t)$ is solution of the Heisenberg equation

$$
i \epsilon Q_{\epsilon}^{\prime}(t)=\left[H(t), Q_{\epsilon}(t)\right]
$$

Since $Q_{\epsilon}(t) Q_{\epsilon}^{\prime}(t) Q_{\epsilon}(t) \equiv 0$, the derivative of $Q_{\epsilon}(t)$ can be written

$$
i Q_{\epsilon}^{\prime}(t)=\left[i\left[Q_{\epsilon}^{\prime}(t), Q_{\epsilon}(t)\right], Q_{\epsilon}(t)\right]
$$

and therefore we rewrite the Heisenberg equation as

$$
\left[H(t)-\epsilon i\left[Q_{\epsilon}^{\prime}(t), Q_{\epsilon}(t)\right], Q_{\epsilon}(t)\right]=0 .
$$

The idea is to find iteratively an approximate solution of the above equation. This can be done even for complex $z \in \Omega$. The leading order in Eq. (2.18) will vanish if we replace $Q_{\epsilon}(z)$ by the approximation $Q_{0}(z)=Q(z)$, since we obviously have $[H(z), Q(z)]=0$. Thus we write formally

$$
Q_{\epsilon}(z)=Q_{0}(z)+\epsilon R_{1}(z, \epsilon),
$$

where $R_{1}(z, \epsilon)$ is a remainder. We are hopeful that it will be of order 1 in $\epsilon$. Inserting this expression for $Q_{\epsilon}(z)$ in Eq. (2.18), we obtain

$$
\left[H(z)-i \epsilon\left[Q_{0}^{\prime}(z), Q_{0}(z)\right]+O\left(\epsilon^{2}\right), Q_{\epsilon}(z)\right]=0,
$$

where ' denotes $d / d z$. Now the leading term of this equation is equal to zero if we choose as a second approximation

$$
Q_{\epsilon}(z)=Q_{1}(z, \epsilon)+\epsilon^{2} R_{2}(z, \epsilon),
$$

where $Q_{1}(z, \epsilon)$ is a spectral projector of the operator

$$
H_{1}(z, \epsilon) \equiv H(z)-i \epsilon\left[Q_{0}^{\prime}(z), Q_{0}(z)\right] .
$$

This definition makes sense since according to perturbation theory, assuming $\epsilon$ to be small, the spectrum of $H_{1}(z, \epsilon)$ is still separated in two disjoint picces, onc of which is bounded. We define $Q_{1}(z, \epsilon)$ as the projector corresponding to the bounded part of the spectrum of $H_{1}(z, \epsilon)$ which tends to $Q_{0}(z)$ as $\epsilon \rightarrow 0$. Again we are confident that $R_{2}(z, \epsilon)$ is of order 1 in $\epsilon$. Now we repeat the same procedure and we are led to the equation 


$$
\left[H(z)-i \epsilon\left[Q_{1}^{\prime}(z, \epsilon), Q_{1}(z, \epsilon)\right]+O\left(\epsilon^{3}\right), Q_{\epsilon}(z)\right]=0 .
$$

Similarly, the leading order will be set to zero by the choice

$$
Q_{\epsilon}(z)=Q_{2}(z, \epsilon)+\epsilon^{2} R_{3}(z, \epsilon),
$$

where $Q_{2}(z, \epsilon)$ is a well-defined spectral projector of

$$
H_{2}(z, \epsilon) \equiv H(z)-i \epsilon\left[Q_{1}^{\prime}(z, \epsilon), Q_{1}(z, \epsilon)\right],
$$

when $\epsilon$ is small and $R_{3}(z, \epsilon)$ is a remainder. We can repeat the whole process as many times as we wish, which provides us with a hierarchy of Hamiltonians whose spectral projectors should solve the Heisenberg equation to an increasing accuracy. We define then an iterative scheme starting with $H_{0}(z) \equiv H(z)$ by the equations

$$
H_{q}(z, \epsilon)=H(z)-\epsilon K_{q-1}(z, \epsilon),
$$

with

$$
K_{q-1}(z, \epsilon)=i\left[Q_{q-1}^{\prime}(z, \epsilon), Q_{q-1}(z, \epsilon)\right]
$$

$Q_{q-1}(z, \epsilon)$ being the spectral projector associated with the bounded part of the spectrum of $H_{q-1}(z, \epsilon)$. As we shall see, this iterative scheme is generally not convergent, as those of Refs. 6-8. We now show how to use effectively this iterative method. In the sequel we drop the $\epsilon$ dependence in the notation for quantities like $H_{q}, Q_{q}$ etc.

Proposition 2.1: Let hypothesis III be true. Let $a, b$, and $c$ be constants such that for all integers $p$, all $z^{\prime} \in D(z, \eta)$

$$
\begin{gathered}
\text { (i) }\left\|R_{0}^{(p)}\left(z^{\prime}, \lambda\right)\right\|=\left\|\frac{d^{p}}{d z^{\prime p}} R_{0}\left(z^{\prime}, \lambda\right)\right\|<a c^{p} \frac{p !}{(1+p)^{2}}, \quad \lambda \in \Gamma, \\
\text { (ii) }\left\|K_{0}^{(p)}\left(z^{\prime}\right)\right\|<b c^{p} \frac{p !}{(1+p)^{2}}, \quad \lambda \in \Gamma .
\end{gathered}
$$

If $\epsilon$ is small enough, $\epsilon<\epsilon^{*}$ with $\epsilon^{*}$ given by Eq. (2.32), then there exists a constant $d$, given by Eq. (2.50), such that

$$
\left\|K_{q}^{(p)}\left(z^{\prime}\right)-K_{q-1}^{(p)}\left(z^{\prime}\right)\right\|<b \epsilon^{q} d^{q} c^{p+q} \frac{(p+q) !}{(1+p)^{2}}
$$

for all $z^{\prime} \in D(z, \eta)$, all integers $p$ and $q$ such that

$$
p+q<\left[\frac{1}{e c d \epsilon}\right] \equiv N^{*} .
$$

Here $[x]$ is the integer part of $x$ and $e$ is the basis of the neperian logarithm. Moreover for all $z^{\prime} \in D(z, \eta)$ and $p+q \leqslant N^{*}$

$$
\left\|K_{q}^{(p)}\left(z^{\prime}\right)\right\|<2 b c^{p} \frac{p !}{(1+p)^{2}} .
$$

Let us briefly comment on the content of this proposition. If we consider the difference between two consecutive terms of the sequence $\left\{H_{q}\right\}_{q>0}$ we obtain 


$$
H_{q+1}(z)-H_{q}(z)=-\epsilon\left(K_{q}(z)-K_{q-1}(z)\right)
$$

Hence, by the above proposition, we have, roughly speaking, a behavior of the type

$$
\left|H_{q+1}(z)-H_{q}(z)\right| \simeq \epsilon^{q}(d c)^{q} q !
$$

where $d c$ is constant, provided $q \leqslant N^{*}$. If we forget about this restriction, we see that we have the typical behavior of asymptotic series, converging at the first steps and then diverging as $q \rightarrow \infty$ for fixed $\epsilon$. The natural idea, which we shall use in Sec. II B, is to stop the sequence at the step where the difference between consecutive terms is the least. Using Stirling formula, we get that the optimal truncation is at the $N^{* \text { th }}$ step, $N^{*}=[1 / e c d \epsilon]$ yielding an exponentially small difference in $1 / \epsilon$ [see Eq. (2.78)]. These behaviors are common to the schemes used in Refs. 5, 7, and 8.

Proof: Let $\alpha$ be the best constant such that for all integers $n$

$$
\sum_{\substack{n_{1}, n_{2} \in \mathrm{N} \\ n_{1}+n_{2}=n}} \frac{1}{\left(1+n_{1}\right)^{2}} \frac{1}{\left(1+n_{2}\right)^{2}}<\frac{\alpha}{(1+n)^{2}}
$$

Let $\epsilon$ be small enough so that $2 \epsilon a b<1$ and

$$
\delta(\epsilon):=\frac{1}{1-2 \epsilon a b}<\infty
$$

We define $\epsilon^{*}$ as the largest $\epsilon$ so that

$$
\sum_{k>1}\left(2 \delta(\epsilon) \epsilon \alpha^{2} a b\right)^{k} \leqslant \alpha
$$

where $\alpha$ is the constant of Eq. (2.30). From now on $0<\epsilon<\epsilon^{*}$ and $\delta=\delta\left(\epsilon^{*}\right)>1$ is independent of $\epsilon$. Let us do the first iteration. Since $2 a b \epsilon<1$ we can define, for all $z^{\prime} \in D(z, \eta)$ and all $\lambda \in \Gamma$,

$$
\left(\mathbb{I}-\epsilon R_{0} K_{0}\right)^{-1}=\sum_{k>0}\left(\epsilon R_{0} K_{0}\right)^{k}
$$

and

$$
\left\|\left(\mathbb{I}-\epsilon R_{0} K_{0}\right)^{-1}\right\| \leqslant \frac{1}{1-a b \epsilon} \leqslant \delta .
$$

Since $R_{0}$ and $K_{0}$ are bounded holomorphic operators on $D(z, \eta)$, the same is true for $\left(\mathbb{I}-\epsilon R_{0} K_{0}\right)^{-1}$ if $\lambda \in \Gamma$.

Lemma 2.1: For all $p \in \mathbf{N}$ and $z^{\prime} \in D(z, \eta)$

$$
\left\|\frac{d^{p}}{d z^{\prime p}}\left(\mathbb{\mathbb { H }}-\epsilon R_{0}\left(z^{\prime}, \lambda\right) K_{0}\left(z^{\prime}\right)\right)^{-1}\right\| \leqslant \delta \frac{p ! c^{p}}{(1+p)^{2}} .
$$

Proof: For $\lambda \in \Gamma$ we have using Eq. (2.30)

$$
\left\|\left(R_{0} K_{0}\right)^{(p)}\right\|<a b \sum_{k=0}^{p}\left(\begin{array}{l}
p \\
k
\end{array}\right) c^{p} \frac{k !}{(1+k)^{2}} \frac{(p-k) !}{(1+(p-k))^{2}} \leqslant \alpha a b c^{p} \frac{p !}{(1+p)^{2}} .
$$

Then we use the formula 


$$
\begin{aligned}
\frac{d^{p}}{d z^{p}}(\mathbb{I}-A(z))^{-1}= & \sum_{k=1}^{p} \sum_{\substack{n_{1}>1, \ldots, n_{k}>1 \\
n_{1}+\cdots+n_{k}=p}}\left(\begin{array}{c}
p \\
n_{1} \cdots n_{k}
\end{array}\right)(\mathbb{I}-A(z))^{-1} A(z)^{\left(n_{1}\right)} \\
& \times(\mathbb{I}-A(z))^{-1} A(z)^{\left(n_{2}\right) \cdots A(z)^{\left(n_{k}\right)}(\mathbb{I}-A(z))^{-1}}
\end{aligned}
$$

and we get

$$
\| \frac{d^{p}}{d z^{p}}\left(\mathbb{I}-\epsilon R_{0} K_{0}\right)^{-1}||<p ! c^{p} \delta \sum_{k=1}^{p}(\epsilon \delta \alpha a b)^{k} \alpha^{k-1} \frac{1}{(1+p)^{2}},
$$

where the factor $\alpha^{k-1}$ comes from the summation over $n_{1}, \ldots, n_{k}$, which is done iteratively as follows

$$
\begin{gathered}
\sum_{\substack{n_{1}>1, \ldots, n_{k}>1 \\
n_{1}+\cdots+n_{k}=p}} \frac{1}{\left(1+n_{1}\right)^{2}} \frac{1}{\left(1+n_{2}\right)^{2}} \cdots \frac{1}{\left(1+n_{k}\right)^{2}} \\
=\sum_{\substack{n_{1}>1, \ldots, n_{k-2}>1, m>2 \\
n_{1}+\cdots+n_{k-2}+m=p}} \frac{1}{\left(1+n_{1}\right)^{2}} \cdots \frac{1}{\left(1+n_{k-2}\right)^{2}} \sum_{\substack{n_{k-1}>1, n_{k}>1 \\
n_{k-1}+n_{k}=m}} \frac{1}{\left(1+n_{k-1}\right)^{2}} \frac{1}{\left(1+n_{k}\right)^{2}} \\
\leqslant \alpha \sum_{\substack{n_{1}>1, n_{2}>1 \ldots, n_{k-1}>1 \\
n_{1}+n_{2} \cdots+n_{k-1}=p}} \frac{1}{\left(1+n_{1}\right)^{2}} \frac{1}{\left(1+n_{2}\right)^{2}} \cdots \frac{1}{\left(1+n_{k-1}\right)^{2}}
\end{gathered}
$$

using Eq. (2.30). Since $\epsilon<\epsilon^{*}$

$$
\| \frac{d^{p}}{d z^{p}}\left(\mathbb{I}-\epsilon R_{0} K_{0}\right)^{-1}||<\delta \frac{p ! c^{p}}{(1+p)^{2}}
$$

We can define for $\lambda \in \Gamma$

$$
R_{1}(z, \lambda)=\left(\mathbb{I}-\epsilon R_{0}(z, \lambda) K_{0}(z)\right)^{-1} R_{0}(z, \lambda),
$$

which is the resolvent of $H_{0}(z)-\epsilon K_{0}(z)$ at $\lambda$. Using lemma (2.1), Leibniz formula and Eq. (2.30) we have for $\lambda \in \Gamma$

$$
\| \frac{d^{p}}{d z^{p}} R_{1}(z, \lambda)||<\alpha \delta a c^{p} \frac{p !}{(1+p)^{2}} .
$$

The next step is to estimate $\left\|K_{1}^{(p)}(z)-K_{0}^{(p)}(z)\right\|$. We have

$$
\begin{aligned}
& \left\|K_{1}^{(p)}-K_{0}^{(p)}\right\| \leqslant\left\|\left(\left(Q_{1}^{\prime}-Q_{0}^{\prime}\right) Q_{1}\right)^{(p)}\right\|+\left\|\left(Q_{0}^{\prime}\left(Q_{1}-Q_{0}\right)\right)^{(p)}\right\|+\left\|\left(\left(Q_{1}-Q_{0}\right) Q_{1}^{\prime}\right)^{(p)}\right\| \\
& +\left\|\left(Q_{0}\left(Q_{1}^{\prime}-Q_{0}^{\prime}\right)\right)^{(p)}\right\| .
\end{aligned}
$$

Since all $\lambda \in \Gamma$ are in the resolvent set of $H_{1}$ we can write 


$$
Q_{1}(z)-Q_{0}(z)=-\frac{1}{2 \pi i} \oint_{\Gamma}\left(R_{1}(z, \lambda)-R_{0}(z, \lambda)\right) d \lambda=-\frac{\epsilon}{2 \pi i} \oint_{\Gamma} R_{0}(z, \lambda) K_{0}(z) R_{1}(z, \lambda) d \lambda
$$

Using Leibniz formula we have

$$
\left\|\left(R_{0} K_{0} R_{1}\right)^{(p)}\right\| \leqslant \alpha^{3} \delta a^{2} b c^{p} \frac{p !}{(1+p)^{2}}
$$

and therefore

$$
\left\|\left(Q_{1}-Q_{0}\right)^{(p)}\right\| \leqslant \frac{|\Gamma|}{2 \pi} \epsilon \alpha^{3} \delta a^{2} b c^{p} \frac{p !}{(1+p)^{2}},
$$

where $|\Gamma|$ is the length of the path $\Gamma$. Using again a Riesz formula for the projectors $Q_{0}$ and $Q_{1}$ and the estimates for $R_{0}$ and $R_{1}$, we get

$$
\left\|Q_{0}^{(p)}\right\| \leqslant \frac{|\Gamma|}{2 \pi} a c^{p} \frac{p !}{(1+p)^{2}}
$$

and

$$
\left\|Q_{1}^{(p)}\right\| \leqslant \frac{|\Gamma|}{2 \pi} \alpha \delta a c^{p} \frac{p !}{(1+p)^{2}}
$$

Lemma 2.2: (Ref. 5) Let $A$ and $B$ be two bounded operators such that

$$
\left\|A^{(p)}\right\| \leqslant a c^{k+p} \frac{(k+p) !}{(1+p)^{2}}
$$

and

$$
\left\|B^{(p)}\right\| \leqslant b c^{l+p} \frac{(l+p) !}{(1+p)^{2}}
$$

Then

$$
\left\|(A B)^{(p)}\right\| \leqslant \alpha a b c^{k+l+p} \frac{(k+l+p) !}{(1+p)^{2}}
$$

By Lemma 2.2

$$
\left\|\left(\left(Q_{1}^{\prime}-Q_{0}^{\prime}\right) Q_{1}\right)^{(p)}\right\| \leqslant \epsilon\left(\frac{|\Gamma|}{2 \pi}\right)^{2} a^{3} b \delta^{2} \alpha^{5} c^{p+1} \frac{(p+1) !}{(1+p)^{2}}
$$

and

$$
\left\|\left(Q_{0}^{\prime}\left(Q_{1}-Q_{0}\right)\right)^{(p)}\right\| \leqslant \epsilon\left(\frac{|\Gamma|}{2 \pi}\right)^{2} a^{3} b \delta \alpha^{4} c^{p+1} \frac{(p+1) !}{(1+p)^{2}}
$$

We introduce 


$$
d:=4 \delta^{3} \alpha^{6}\left(\frac{|\Gamma|}{2 \pi}\right)^{2} a^{3}
$$

Therefore we can write, since $\alpha>1$ and $\delta>1$,

$$
\left\|K_{1}^{(p)}-K_{0}^{(p)}\right\|<\epsilon b d c^{p+1} \frac{(p+1) !}{(1+p)^{2}}
$$

We estimate $\left\|K_{1}^{p}\right\|$ for $1+p<N^{*}$.

$$
\begin{aligned}
\left\|K_{1}^{(p)}\right\| & <\left\|K_{1}^{(p)}-K_{0}^{(p)}\right\|+\left\|K_{0}^{(p)}\right\| \\
& <\epsilon b d c^{p+1} \frac{(p+1) !}{(1+p)^{2}}+b c^{p} \frac{p !}{(1+p)^{2}} \\
& =b c^{p} \frac{p !}{(1+p)^{2}}(1+\epsilon d c(p+1)) \\
& <b c^{(p)} \frac{p !}{(1+p)^{2}} \sum_{k>0}\left(\epsilon c d N^{*}\right)^{k} \\
& <b c^{p} \frac{p !}{(1+p)^{2}} \sum_{k>0} e^{-k} \\
& =b \frac{e}{e-1} c^{p} \frac{p !}{(1+p)^{2}} \\
& <2 b c^{p} \frac{p !}{(1+p)^{2}} .
\end{aligned}
$$

With these last estimates we have finished all estimates for the first iteration.

We do again all estimates for the next iteration. For all $\lambda \in \Gamma$ and all $z^{\prime} \in D(z, \eta)$ we have by our hypothesis on $\epsilon$ [see Eq. (2.31)],

$$
\left\|\left(\mathbb{I}-\epsilon R_{0} K_{1}\right)^{-1}\right\|<\sum_{k>0}\left(\left\|\epsilon R_{0} K_{1}\right\|\right)^{k} \leqslant \delta
$$

and

$$
\left\|\left(R_{0} K_{1}\right)^{(p)}\right\|<2 b a \alpha c^{p} \frac{p !}{(1+p)^{2}}
$$

As for Lemma 2.1 we get with Eq. (2.32),

$$
\begin{aligned}
\| \frac{d^{p}}{d z^{p}}\left(\mathbb{I}-\epsilon R_{0} K_{1}\right)^{-1} \mid & \mid<p ! c^{p} \delta \sum_{k=1}^{p}(2 \epsilon \delta \alpha a b)^{k} \alpha^{k-1} \frac{1}{(1+p)^{2}} \\
& <p ! c^{p} \delta \frac{1}{(1+p)^{2}} \frac{1}{\alpha} \sum_{k=1}^{p}\left(2 \epsilon \delta \alpha^{2} a b\right)^{k}
\end{aligned}
$$




$$
<p ! c^{p} \delta \frac{1}{(1+p)^{2}}
$$

The resolvent of $H_{2}(z)=H(z)-\epsilon K_{1}(z)$ is given at $\lambda \in \Gamma$ by

$$
R_{2}(z, \lambda)=\left(\mathbb{I}-\epsilon R_{0}(z) K_{1}(z)\right)^{-1} R_{0}(z)
$$

and thus for $1+p<N^{*}$

$$
\| \frac{d^{p}}{d z^{p}} R_{2}(z, \lambda)|| \leqslant \alpha \delta a c^{p} \frac{p !}{(1+p)^{2}} .
$$

Then we estimate $\left\|\left(K_{2}-K_{1}\right)^{(p)}\right\|$ as above. For $\lambda \in \Gamma$

$$
\begin{aligned}
Q_{2}(z)-Q_{1}(z) & =-\frac{1}{2 \pi i} \oint_{\Gamma}\left(R_{2}(z, \lambda)-R_{1}(z, \lambda)\right) d \lambda \\
& =-\frac{\epsilon}{2 \pi i} \oint_{\Gamma} R_{1}(z, \lambda)\left(K_{1}(z)-K_{0}(z)\right) R_{2}(z, \lambda) d \lambda
\end{aligned}
$$

We get for $p+1<N^{*}$

$$
\left\|\left(Q_{2}-Q_{1}\right)^{(p)}\right\| \leqslant \frac{|\Gamma|}{2 \pi} \epsilon^{2} b d \alpha^{4} \delta^{2} a^{2} c^{p+1} \frac{(p+1) !}{(1+p)^{2}}
$$

Thus

$$
\left\|\left(\left(Q_{2}^{\prime}-Q_{1}^{\prime}\right) Q_{2}\right)^{(p)}\right\|<\epsilon^{2}\left(\frac{|\Gamma|}{2 \pi}\right)^{2} a^{3} b d \delta^{3} \alpha^{6} c^{p+2} \frac{(p+2) !}{(1+p)^{2}}
$$

and

$$
\left\|\left(Q_{1}^{\prime}\left(Q_{2}-Q_{1}\right)\right)^{(p)}\right\|<\epsilon^{2}\left(\frac{|\Gamma|}{2 \pi}\right)^{2} a^{3} b d \delta^{3} \alpha^{6} c^{p+2} \frac{(p+2) !}{(1+p)^{2}}
$$

Therefore

$$
\left\|K_{2}^{(p)}-K_{1}^{(p)}\right\| \leqslant \epsilon^{2} b d^{2} c^{p+2} \frac{(p+2) !}{(1+p)^{2}}
$$

and for all $2+p<N^{*}$

$$
\begin{aligned}
\left\|K_{2}^{(p)}\right\| & <\left\|K_{2}^{(p)}-K_{1}^{(p)}\right\|+\left\|K_{1}^{(p)}-K_{0}^{(p)}\right\|+\left\|K_{0}^{(p)}\right\| \\
& <b c^{p} \frac{p !}{(1+p)^{2}}\left(1+\epsilon d c(p+2)+\epsilon^{2} d^{2} c^{2}(p+2)^{2}\right) \\
& <b \frac{e}{e-1} c^{p} \frac{p !}{(1+p)^{2}}
\end{aligned}
$$




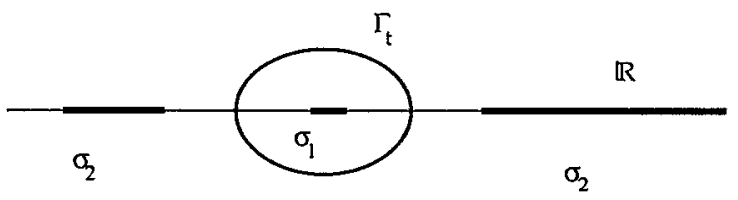

FIG. 1. The contour $\Gamma_{t}$ for $t \in \mathbf{R}$.

$$
<2 b c^{p} \frac{p !}{(1+p)^{2}}
$$

We can iterate this procedure since all estimates are reproduced as long as $p+q \leqslant N^{*}$ is satisfied.

\section{B. Superadiabatic evolution}

We now construct the superadiabatic evolution. Since we consider later on a scattering situation we add to the hypothesis I and II the hypothesis

(IV) Behavior for large times: There exist two self-adjoint operators $H^{1}$ and $H^{-}$bounded from below and defined on $D$, and an integrable function $b(t)$ on $\mathbb{R}$, behaving like $1 /|t|^{1+\alpha}$ at infinity for some $\alpha>0$, such that

$$
\sup _{|s|<r}\left\|\left(H(t+i s)-H^{+}\right) \varphi\right\| \leqslant b(t)\left(\|\varphi\|+\left\|H^{+} \varphi\right\|\right), \quad t>0
$$

and

$$
\sup _{|s|<r}\left\|\left(H(t+i s)-H^{-}\right) \varphi\right\|<b(t)\left(\|\varphi\|+\left\|H^{-} \varphi\right\|\right), \quad t<0 .
$$

Let $t$ be some point of the real axis. Since $H(t)$ is self-adjoint we have for all $\lambda$ in the resolvent set of $H(t)$, with $\operatorname{dist}(\lambda, \sigma(t))=$ distance of $\lambda$ to the set $\sigma(t)$,

$$
\left\|R_{0}(t, \lambda)\right\|=\frac{1}{\operatorname{dist}(\lambda, \sigma(t))}
$$

where $\sigma(t)$ is the spectrum of $H(t)$. For each $t$ we choose $\Gamma_{t}$ as in Fig. 1, so that for any $\lambda \in \Gamma_{t}$

$$
\operatorname{dist}(\lambda, \sigma(t)) \geqslant \frac{1}{2} \operatorname{dist}\left(\sigma_{1}(t), \sigma_{2}(t)\right) \geqslant \frac{g}{2}
$$

It follows from condition II and Eq. (2.19) in Ref. 4 that we may choose the width $r$ of the neighborhood $\Omega$ so that for each $z \in D(t, r)=\{z \in \mathbb{C}:|z-t| \leqslant r\}$, and all $t \in \mathbb{R}$

$$
\left\|R_{0}(z, \lambda)\right\| \leqslant 2\left\|R_{0}(t, \lambda)\right\| \leqslant \frac{4}{\operatorname{dist}\left(\sigma_{1}(t), \sigma_{2}(t)\right)} \leqslant \frac{4}{g} \forall \lambda \in \Gamma_{t}
$$

hence hypothesis III is satisfied. Moreover, by lemma 2.2 in Ref. 4 that there exists a constant $C^{\prime}$ such that for all $z \in D(t, r)$ and all $t \in \mathbb{R}$

$$
\left\|K_{0}(z)\right\| \leqslant C^{\prime} b(t)
$$


with $b(t)$ the integrable function of condition IV. Using Cauchy formula we get for all $z$ $\in D(t, r / 2)$,

$$
\left\|R_{0}^{(p)}(z, \lambda)\right\| \leqslant \frac{4}{g} p !\left(\frac{2}{r}\right)^{p}
$$

and

$$
\left\|K_{0}^{(p)}(z)\right\| \leqslant C^{\prime} b(t) p !\left(\frac{2}{r}\right)^{p} .
$$

Therefore we can apply proposition 2.1 with $c=8 / r, a=4 / g$, and $b=C^{\prime} b(t)$. Hence there exists $\epsilon^{*}$ independent of $t$ such that for all $\epsilon<\epsilon^{*}$ the iteration scheme is well defined up to order $N^{*}=\left[(e c d \epsilon)^{-1}\right]$ with

$$
d=\frac{\delta^{3} \alpha^{6}}{\pi^{2}} L^{2} a^{3}=64 \frac{\delta^{3} \alpha^{6}}{\pi^{2}} \frac{L^{2}}{g^{3}}=O\left(\frac{L^{2}}{g^{3}}\right),
$$

where $L$ is the supremum of the length of the paths $\Gamma_{t}$. We set for all $t \in \mathbb{R}$

$$
H_{*}(t):=H_{N *}(t)=H(t)-\epsilon K_{N^{*-1}}(t)
$$

and

$$
Q_{*}(t):=-\frac{1}{2 \pi i} \oint_{\Gamma_{t}} R_{N^{*}}(t, \lambda) d \lambda .
$$

Since $\left\|K_{N^{*}-1}(t)\right\|=O(b(t))$, we have

$$
\lim _{t \rightarrow \pm \infty}\left\|Q_{*}(t)-Q(t)\right\|=0 .
$$

The superadiabatic evolution is defined by the equation

$$
i \epsilon \frac{\partial}{\partial t} V_{*}(t, s)=\left(H_{*}(t)+\epsilon i\left[Q_{*}^{\prime}(t), Q_{*}(t)\right]\right) V_{*}(t, s), \quad V_{*}(s, s)=\mathbb{I}
$$

Theorem 2.1: Under the hypotheses I and II, there exists $\epsilon^{*}$, a positive constant $\tau$, and a positive function $\bar{b}(t)$ such that for all $t, s \in \mathbb{R}$

$$
Q_{*}(t) V_{*}(t, s)=V_{*}(t, s) Q_{*}(s)
$$

and

$$
\left\|U(t, s)-V_{*}(t, s)\right\| \leqslant\left|\int_{s}^{t} \bar{b}\left(t^{\prime}\right) d t^{\prime}\right| \exp \left(-\tau \epsilon^{-1}\right) .
$$

If hypothesis IV holds as well

$$
\int_{-\infty}^{\infty} \bar{b}(t) d t<\infty
$$

Proof: The first part of the theorem can be found in Ref. 11 Chap. 4. Let us prove the last part. We consider the expression 


$$
\begin{aligned}
i \epsilon \frac{\partial}{\partial t}\left(V_{*}^{-1}(t, s) U(t, s)\right) & =V_{*}^{-1}(t, s)\left(-H_{*}(t)-\epsilon K_{N^{*}}(t)+H(t)\right) V_{*}(t, s)\left(V_{*}^{-1}(t, s) U(t, s)\right) \\
& =\epsilon V_{*}^{-1}(t, s)\left(K_{N^{*}-1}(t)-K_{N^{*}}(t)\right) V_{*}(t, s)\left(V_{*}^{-1}(t, s) U(t, s)\right) .
\end{aligned}
$$

From Proposition 2.1 and Stirling formula we have

$$
\begin{aligned}
\left\|K_{N^{*-1}}(t)-K_{N^{*}}(t)\right\| & <C^{\prime} b(t) \epsilon^{N^{*}} d^{N^{*}} c^{N^{*}} N^{* !} \\
& =O(b(t))\left(\epsilon d c N^{*}\right)^{N^{*}} e^{-N^{*}} \sqrt{N^{*}} \\
& =O(b(t)) e^{-(2-\beta) N^{*}} \\
& \equiv \bar{b}(t) \exp \left(-\tau \epsilon^{-1}\right)
\end{aligned}
$$

where $\beta$ is any small positive number, $\bar{b}(t)=O(b(t))$ and

$$
0<\tau=\frac{2-\beta}{e c d}=O\left(\frac{g^{3}}{L^{2}}\right)
$$

Hence it follows that

$$
\left\|V_{*}^{-1}(t, s) U(t, s)-\mathbb{I}\right\| \leqslant\left|\int_{s}^{t} \bar{b}\left(t^{\prime}\right) d t^{\prime}\right| \exp \left(-\tau \epsilon^{-1}\right) .
$$

Remarks: (1) If the bounded part $\sigma_{1}(t)$ of the spectrum has a length which is uniformly bounded, then the decay rate $\tau=O(g)$ when $g$ becomes large. (2) In general, the projectors $Q_{*}(t)$ are a distance of order $\epsilon$ away from the spectral projectors $Q(t)$ of the Hamiltonian. But if $H(t)$ has its $n$ first derivatives equal to zero at $t=t_{0}$, then we easily see that

$$
Q_{*}\left(t_{0}\right)=Q\left(t_{0}\right)+O\left(\epsilon^{n+1}\right)
$$

(3) It follows from this theorem that a solution of the Schrödinger equation initially in the spectral subspace $Q(s) \mathscr{H}$ will follow this subspace up to an error of order $\epsilon|t-s|$. Nevertheless, there exist projectors $Q_{*}(t)$, close to $Q(t)$, such that a solution initially in $Q_{*}(s) \mathscr{H}$ will be confined to the subspace $Q_{*}(t) \mathscr{H}$, for exponentially long times. Moreover, if hypothesis IV holds, this solution will be in $Q_{*}(t) \mathscr{H}$ up to an exponentially small correction, and this for any time $t$. As a consequence, if we take $s=-\infty, t=+\infty$ and use the fact that $H_{*}$ and $H$ coincide at infinity, we can see that the transition probability from $Q(-\infty) \mathscr{H}$ to $(\mathbb{I}-\stackrel{Q}{*}(+\infty)) \mathscr{H}$ is of order $\exp (-\tau / \epsilon)$. Note also that we obtain the physically expected behavior of the transition probability as a function of the gap $g$ since $\tau=O(g)$.

\section{ADIABATIC TRANSITION PROBABILITY}

Let $H(t)$ be a time-dependent self-adjoint operator satisfying the hypothesis I, II, IV, and

(V) Existence of complex eigenvalue crossings: The part of the spectrum $\sigma_{1}(t)$ consists of two separated eigenvalues $e_{1}(t)<e_{2}(t)$ for all $t \in \mathbb{R}$ and there exists $z^{*} \in \Omega$, Im $z^{*}>0$, such that the energy-levels $e_{1}$ and $e_{2}$ have analytic (multivalued) continuations in $\Omega \backslash\left\{z^{*}, z^{*}\right\}$ and are equal at $z^{*}$ and $\bar{z}^{*}$. At the eigenvalue-crossings $z^{*}$ and $\bar{z}^{*}$ the energy-levels $e_{1}$ and $e_{2}$ have a square-root-type singularity. 


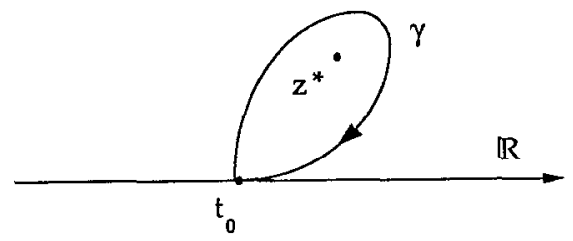

FIG. 2. The loop $\gamma$ encircling the eigenvalue crossing point $z^{*}$.

Remark: It is implicitly assumed that the parts of spectrum $\sigma_{1}(z)$ and $\sigma_{2}(z)$ are disjoint for any $z \in \Omega$.

Let $P_{i}(t), i=1,2$ be the spectral projector associated with $e_{i}(t), i=1,2$. We study the normalized solution $\varphi_{\epsilon}(t)$ of the Schrödinger equation

$$
i \epsilon \frac{\partial}{\partial t} \varphi_{\epsilon}(t)=H(t) \varphi_{\epsilon}(t), \varphi_{\epsilon}(0)=\varphi^{*} \in D
$$

subject to the boundary condition

$$
\lim _{t \rightarrow-\infty}\left\|P_{1}(t) \varphi_{\epsilon}(t)\right\|=1
$$

when $t \rightarrow+\infty$. The boundary condition means that the system is prepared at time $t \rightarrow-\infty$ in an eigenstate of energy $e_{1}(-\infty)$ of $H(-\infty)=H^{-}$. We compute the transition probability

$$
\mathscr{P}_{21}(\epsilon)=\lim _{t \rightarrow \infty}\left\|P_{2}(t) \varphi_{\epsilon}(t)\right\|^{2},
$$

which gives the probability to measure the energy $e_{2}(+\infty)$ at time $t=+\infty$. We can obtain an asymptotic formula for $\mathscr{P}_{21}(\epsilon)$ when the distance between $\sigma_{1}(t)=\left\{e_{1}(t), e_{2}(t)\right\}$ and the rest of the spectrum $\sigma_{2}(t)$ is large enough, i.e., when $g$ is large enough. To do this we need, as in our previous papers 1 and 2 , an additional hypothesis. Let us make it explicit and give the formula for $\mathscr{P}_{21}(\epsilon)$.

Let $\gamma$ be a loop starting at $t_{0}$ as in Fig. 2. Here $t_{0}$ is any fixed point of $\mathbb{R}$. Let $\psi_{1}\left(t_{0}\right)$ and $\psi_{2}\left(t_{0}\right)$ be two normalized eigenvectors of $H\left(t_{0}\right)$,

$$
H\left(t_{0}\right) \psi_{j}\left(t_{0}\right)=e_{j}\left(t_{0}\right) \psi_{j}\left(t_{0}\right), \quad j=1,2,
$$

whose phases are fixed by imposing the condition

$$
\left\langle\psi_{j}(t) \mid \frac{d}{d t} \psi_{j}(t)\right\rangle \equiv 0, \quad j=1,2 \quad \forall t \in \mathbb{R} .
$$

We analytically continue $e_{1}\left(t_{0}\right)$ and $\psi_{1}\left(t_{0}\right)$ along the path $\gamma$ and we denote by $\widetilde{e}_{1}\left(t_{0}\right)$ and $\widetilde{\psi}_{1}\left(t_{0}\right)$ the result of the analytic continuation when we come back to $t_{0}$. By hypothesis $\mathrm{V}$ we have

$$
\tilde{e}_{1}\left(t_{0}\right)=e_{2}\left(t_{0}\right), \quad \widetilde{\psi}_{1}\left(t_{0}\right)=e^{-i \theta_{21}\left(t_{0} \mid \gamma\right)} \psi_{2}\left(t_{0}\right),
$$

where $\theta_{21}\left(t_{0} \mid \gamma\right)$ is in general a complex phase. We also define $\int_{\gamma} e_{1}(z) d z$ as the integral of the analytic continuation of $e_{1}$ along $\gamma$. Both quantities $\theta_{21}\left(t_{0} \mid \gamma\right)$ and $\int_{\gamma} e_{1}(z) d z$ depend only on the homotopy class of the loop $\gamma$ based at $t_{0}$. Moreover, $\operatorname{Im} \theta_{21}\left(t_{0} \mid \gamma\right)$ and $\operatorname{Im} \int_{\gamma} e_{1}(z) d z$ do not depend on the choice of $t_{0}$ and the choice of the phase of $\psi_{2}\left(t_{0}\right)$. By analytic continuation we also define the multivalued function 


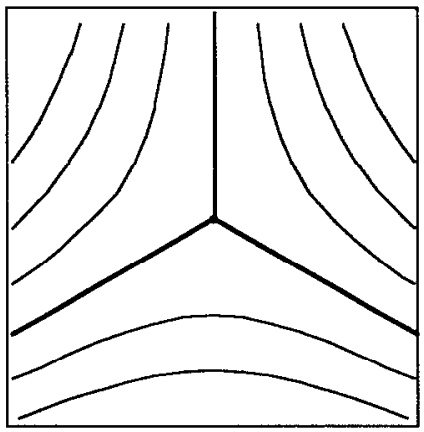

FIG. 3. The level lines of $\operatorname{Im} \Delta_{12}(z)$ near $z^{*}$.

$$
\Delta_{12}(z)=\int_{t_{0}}^{z}\left(e_{1}\left(z^{\prime}\right)-e_{2}\left(z^{\prime}\right)\right) d z^{\prime}
$$

on $\Omega \backslash\left\{z^{*}, z^{*}\right\}$. The structure of the level lines of $\operatorname{Im} \Delta_{12}(z)$, near $z^{*}$ is as in Fig. 3. There are exactly three level lines which meet at $z^{*}$. They are called critical. It is not difficult to show that the three critical lines have no intersection point in $\Omega \backslash\left\{z^{*}, \bar{z}^{*}\right\}$, and that they are always in the upper half plane. We have a similar situation at $\bar{z}^{*}$. The next hypothesis is a global condition on the behavior of the critical lines.

(VI) Existence of infinite critical lines: There exist two critical lines of $\Delta_{12}(z)$ in $\Omega$, one going from $z^{*}$ to $-\infty$, and the other one going from $z^{*}$ to $+\infty$.

The condition VI is analyzed in details in Ref. 1. In particular, a geometrical formulation of this condition is given. We can now state our main result.

Theorem 3.1: Let $H(t)$ be a self-adjoint operator analytic in $t$, satisfying conditions I, II, IV, V, and VI. Let $\varphi_{\epsilon}(t)$ be a normalized solution of the Schrödinger equation

$$
i \epsilon \frac{\partial}{\partial t} \varphi_{\epsilon}(t)=H(t) \varphi_{\epsilon}(t)
$$

such that

$$
\lim _{t \rightarrow-\infty}\left\|P_{1}(t) \varphi_{\epsilon}(t)\right\|=1
$$

If $\inf { }_{t \in \mathbf{R}} \operatorname{dist}\left(\sigma_{1}(t), \sigma_{2}(t)\right) /\left|e_{1}(t)-e_{2}(t)\right|$ is large enough, then

$$
\mathscr{P}_{21}(\epsilon)=\lim _{t \rightarrow \infty}\left\|P_{2}(t) \varphi_{\epsilon}(t)\right\|^{2}=\exp \left(2 \operatorname{Im} \theta_{21}\left(t_{0} \mid \gamma\right)\right) \exp \left(2 \epsilon^{-1} \operatorname{Im} \int_{\gamma} e_{1}(z) d z\right)(1+O(\epsilon))
$$

Remark: The last condition on the spectrum of the Hamiltonian means that the decay rate $\tau$ of theorem 2.1 is larger than $\left|2 \operatorname{Im} \int_{\gamma} e_{1}(z) d z\right|$. Physically this condition means that the probability to make a transition into the spectral subspace of $\sigma_{2}(\infty)$ is smaller than $\mathscr{P}_{21}$.

Moreover, we have an cxplicit formula for $\operatorname{Im} \theta_{21}$ in terms of the matrix elements of the reduced Hamiltonian $Q(t) H(t)$ in the time-dependent two-dimensional subspace $Q(t) \mathscr{H}$. Let $\varphi_{1}(z)$ and $\varphi_{2}(z)$ form a basis of analytic vectors of $Q(z) \mathscr{H}, \forall z \in \Omega$ which are orthonormal when $z=t \in \mathbb{R}$. Without loss of generality we assume that $e_{1}(t)+e_{2}(t) \equiv 0$ so that we can write for $t \in \mathbb{R}$

$$
\left.H(t)\right|_{Q(t) \not P}=\mathbf{B}(t) \cdot \mathbf{S}
$$


in the basis $\left\{\varphi_{1}(t), \varphi_{2}(t)\right\}$ with $s_{j} j=1,2,3$, the spin-1/2 matrices, and with an analytic "magnetic field" $\mathbf{B}(t)$ defined by its components

$$
\begin{gathered}
B_{1}(t)=2 \operatorname{Re}\left\langle\varphi_{1}(t) \mid H(t) \varphi_{2}(t)\right\rangle, \\
B_{2}(t)=-2 \operatorname{Im}\left\langle\varphi_{1}(t) \mid H(t) \varphi_{2}(t)\right\rangle, \\
B_{3}(t)=2\left\langle\varphi_{1}(t) \mid H(t) \varphi_{1}(t)\right\rangle
\end{gathered}
$$

With these definitions, the eigenvalues of $H(t) Q(t)$ are given by the relation

$$
e_{j}(t)=(-1)^{j \frac{1}{2}} \sqrt{\rho(t)}, \quad j=1,2,
$$

where

$$
\rho(t)=\sum_{j=1}^{3} B_{j}^{2}(t)
$$

Proposition 3.1:

$$
\begin{aligned}
\operatorname{Im} \theta_{21}= & \operatorname{Im} \int_{\sigma} \frac{B_{3}(z)\left(B_{1}(z) B_{2}^{\prime}(z)-B_{2}(z) B_{1}^{\prime}(z)\right)}{2 \sqrt{\rho(z)}\left(B_{1}^{2}(z)+B_{2}^{2}(z)\right)} d z+\operatorname{Re} \int_{\sigma}\left(\frac { B _ { 3 } ( z ) } { 2 \sqrt { \rho ( z ) } } \left(\left\langle\varphi_{1} \mid \varphi_{1}^{\prime}\right\rangle(z)\right.\right. \\
& \left.\left.-\left\langle\varphi_{2} \mid \varphi_{2}^{\prime}\right\rangle(z)\right)+\frac{B_{1}(z)+i B_{2}(z)}{2 \sqrt{\rho(z)}}\left\langle\varphi_{1} \mid \varphi_{2}^{\prime}\right\rangle(z)+\frac{B_{1}(z)-i B_{2}(z)}{2 \sqrt{\rho(z)}}\left\langle\varphi_{2} \mid \varphi_{1}^{\prime}\right\rangle(z)\right) d z,
\end{aligned}
$$

where the loop $\sigma$ is based at the origin, encircles $z^{*}$ and contains no zero of $B_{1}^{2}(z)+B_{2}^{2}(z)$ and $\left\langle\varphi_{j} \mid \varphi_{k}^{\prime}\right\rangle(z)$ is the analytic continuation of $\left\langle\varphi_{j}(t) \mid \varphi_{k}^{\prime}(t)\right\rangle, t \in \mathbb{R}$.

Remark: The first term in the above formula coincides with the expression of $\operatorname{Im} \theta_{21}$ given in Ref. 1 for two-level systems but the necessary time dependence of the basis of $Q(t) \mathscr{H}$ induces, in general, supplementary terms. Note that the "magnetic field" $\mathbf{B}(t)$ depends explicitly on the chosen basis in $Q(t) \mathscr{H}$. However, as explained in the Appendix, there exists a natural basis of $Q(t) \mathscr{H}$ given by the usual parallel transport operator [see Eq. (A16)] such that the expression of $\operatorname{Im} \theta_{21}$ in terms of the matrix elements of $H$ in that basis contains no supplementary contributions. But it should be noted that there is, in general, no explicit formula giving the vectors of this particular basis. The proof of that proposition together with an explicit choice of basis vectors having the required properties can be found in the Appendix.

\section{A. Reduction to an effective two-dimenslonal problem}

We derive here an expression for the transition probability $\mathscr{P}_{21}$ which shows that the asymptotics of $\mathscr{P}_{21}$ can be computed by solving an effective two-dimensional problem if $\tau$ of proposition 2.1 is large enough. The exposition follows Sec. IV of Ref. 4 . It is convenient to decompose the superadiabatic cvolution $V_{*}$ into two parts,

$$
V_{*}(t, 0):=W_{*}(t, 0) \Phi_{*}(t, 0),
$$

where $W_{*}(t, 0)$ is defined by

$$
i \frac{\partial}{\partial t} W_{*}(t, 0)=K_{N *}(t) W_{*}(t, 0), \quad W_{*}(0,0)=\mathbb{I}
$$


and leaves the domain $D$ invariant (see Lemma 5.1 in Ref. 4). From now on $s=0$ and we omit this index. The decomposition equation (3.14) is a generalization of the decomposition of the adiabatic evolution into a "parallel transport" $W_{*}(t)$ and a "dynamical phase factor" $\Phi_{*}(t)$. The operator $\Phi_{*}(t)$ is the solution of

$$
i \epsilon \frac{\partial}{\partial t} \Phi_{*}(t)=W_{*}^{-1}(t) H_{*}(t) W_{*}(t) \Phi_{*}(t) \equiv \hat{H}_{*}(t) \Phi_{*}(t), \quad \Phi_{*}(0)=\mathbb{I} .
$$

The evolution $W_{*}$ is compatible with the decomposition of $\mathscr{H}$ into

$$
\mathscr{H}=Q_{*}(t) \mathscr{H} \oplus\left(\mathbb{I}-Q_{*}(t)\right) \mathscr{H}
$$

so that we have

$$
Q_{*}(t) W_{*}(t)=W_{*}(t) Q_{*}(0)
$$

and $\Phi_{*}$ satisfies

$$
\left[\Phi_{*}(t), Q_{*}(0)\right]=0
$$

Therefore we can write

$$
U(t)=W_{*}(t) \Phi_{*}(t) A_{*}(t)
$$

with $A_{*}(t)$ satisfying Eq. (2.77). From Eq. (2.78) we see that $A_{*}(t)$ has well-defined limits when $t \rightarrow \pm \infty$. The operator $\hat{H}_{*}(t)$ commutes with $Q_{*}(0)$ and if $\epsilon$ is small enough $Q_{*}(0)$ $=\hat{P}_{1}^{*}(t)+\hat{P}_{2}^{*}(t)$ with

$$
\hat{P}_{j}^{*}(t)-W_{*}^{-1}(t) P_{j}^{*}(t) W_{*}(t), \quad j=1,2,
$$

where $\hat{P}_{j}^{*}(t)$ are spectral projectors of $\hat{H}_{*}(t)$. It is therefore possible to decompose the evolution $\Phi_{*}(t)$ as we did for $U(t)$. We introduce the evolution $\hat{V}_{*}(t)$

$$
i \epsilon \frac{\partial}{\partial t} \hat{V}_{*}(t)=\left(\hat{H}_{*}(t)+i \epsilon \sum_{j=1}^{2} \frac{\partial}{\partial t} \hat{P}_{j}^{*}(t) \hat{P}_{j}^{*}(t)\right) \hat{V}_{*}(t), \quad \hat{V}_{*}(0)=\mathbb{I}
$$

and we set

$$
\Phi_{*}(t):=\hat{V}_{*}(t) \hat{A}_{*}(t)
$$

By construction $\hat{V}_{*}(t)$ is compatible with the decomposition of $Q_{*}(0) \mathscr{H}$ into

$$
Q_{*}(0) \mathscr{H}=\hat{P}_{1}^{*}(t) \mathscr{H} \oplus \hat{P}_{2}^{*}(t) \mathscr{H}
$$

since

$$
\hat{P}_{j}^{*}(t) \hat{V}_{*}(t)=\hat{V}_{*}(t) \hat{P}_{j}^{*}(0), \quad j=1,2
$$

The operator $\hat{A}_{*}(t)$ is the solution of the equation

$$
i \frac{\partial}{\partial t} \hat{A}_{*}(t)=-\left(\hat{V}_{*}^{-1}(t) i\left(\sum_{j=1}^{2} \frac{\partial}{\partial t} \hat{P}_{j}^{*}(t) \hat{P}_{j}^{*}(t)\right) \hat{V}_{*}(t)\right) \hat{A}_{*}(t), \quad \hat{A}_{*}(0)=\mathbb{I} .
$$


Since $\left\|\Sigma_{j=1}^{2}(\partial / \partial t) \hat{P}_{j}^{*}(t) \hat{P}_{j}^{*}(t)\right\|$ is integrable as $t \rightarrow \pm \infty$ the operator $\hat{A}_{*}(t)$ has well-defined limits when $t \rightarrow \pm \infty$. We also have

$$
\lim _{t \rightarrow \pm \infty}\left\|P_{j}^{*}(t)-P_{j}(t)\right\|=0
$$

by Eq. (2.73).

Proposition 3.2: Let $\varphi(t)$ be a normalized solution of

$$
i \epsilon \frac{\partial}{\partial t} \varphi(t)=H(t) \varphi(t)
$$

satisfying the boundary condition

$$
\lim _{t \rightarrow-\infty}\left\|P_{1}(t) \varphi(t)\right\|=1
$$

If $\epsilon$ is small enough, then

$$
\mathscr{P}_{21}=\lim _{t \rightarrow+\infty}\left\|P_{2}(t) \varphi(t)\right\|^{2}=\left\|\hat{P}_{2}^{*}(0) \hat{A}_{*}(\infty) A_{*}(\infty) A_{*}^{-1}(-\infty) \hat{A}_{*}(-\infty) \hat{P}_{1}^{*}(0)\right\|^{2}
$$

Moreover,

$$
A_{*}(\infty) A_{*}^{-1}(-\infty)=\mathbb{I}+\Delta(\infty,-\infty)
$$

and

$$
\|\Delta(\infty,-\infty)\| \leqslant M \exp \left(-\tau \epsilon^{-1}\right),
$$

with $M$ and $\tau$ as in proposition 2.1.

Proof: Let $\varphi(t)$ with $\varphi(0)=\varphi^{*}$ be given. We have [using Eqs. (3.27), (3.21), (3.23), and (3.25)]

$$
\begin{aligned}
1= & \lim _{t \rightarrow-\infty}\left\|P_{1}(t) \varphi(t)\right\| \\
= & \lim _{t \rightarrow-\infty}\left\|P_{1}^{*}(t) W_{*}(t) \Phi_{*}(t) A_{*}(t) \varphi^{*}\right\| \\
= & \lim _{t \rightarrow-\infty}\left\|W_{*}(t) \hat{P}_{1}^{*}(t) \Phi_{*}(t) A_{*}(t) \varphi^{*}\right\| \\
= & \lim _{t \rightarrow-\infty}\left\|\hat{P}_{1}^{*}(t) \hat{V}_{*}(t) \hat{A}_{*}(t) A_{*}(t) \varphi^{*}\right\| \\
= & \lim _{t \rightarrow-\infty}\left\|\hat{V}_{*}(t) \hat{P}_{1}^{*}(0) \hat{A}_{*}(t) A_{*}(t) \varphi^{*}\right\| \\
= & \left\|\hat{P}_{1}^{*}(0) \hat{A}_{*}(-\infty) A_{*}(-\infty) \varphi^{*}\right\| .
\end{aligned}
$$

Therefore we can write

$$
\varphi^{*}=A_{*}^{-1}(-\infty) \hat{A}_{*}^{-1}(-\infty) \psi
$$

with $\psi \in \hat{P}_{1}^{*}(0) \mathscr{H}$. By a computation similar to Eq. (3.28) we have 


$$
\begin{aligned}
\lim _{t \rightarrow \infty}\left\|P_{2}(t) \varphi(t)\right\| & =\lim _{t \rightarrow \infty}\left\|P_{2}^{*}(t) \varphi(t)\right\| \\
& =\left\|\hat{P}_{2}^{*}(0) \hat{A}_{*}(\infty) A_{*}(\infty) \varphi^{*}\right\| \\
& =\left\|\hat{P}_{2}^{*}(0) \hat{A}_{*}(\infty) A_{*}(\infty) A_{*}^{-1}(-\infty) \hat{A}_{*}^{-1}(-\infty) \hat{P}_{1}^{*}(0)\right\|
\end{aligned}
$$

The last assertion follows from proposition 2.1 and from

$$
A_{*}(t)=\mathbb{I}+\int_{0}^{t} V_{*}^{-1}\left(t^{\prime}\right)\left(K_{N^{*-1}}\left(t^{\prime}\right)-K_{N^{*}}\left(t^{\prime}\right)\right) V_{*}\left(t^{\prime}\right) A_{*}\left(t^{\prime}\right) d t^{\prime}
$$

By proposition 3.2 we can write

$$
\begin{aligned}
\hat{P}_{2}^{*}(0) & \hat{A}_{*}(\infty)(\mathbb{I}+\Delta(\infty,-\infty)) \hat{A}_{*}^{-1}(-\infty) \hat{P}_{1}^{*}(0) \\
= & \hat{P}_{2}^{*}(0) \hat{A}_{*}(\infty) \hat{A}_{*}^{1}(-\infty) \hat{P}_{1}^{*}(0) \\
& +\hat{P}_{2}^{*}(0) \hat{A}_{*}(\infty) \Delta(\infty,-\infty) \hat{A}_{*}^{-1}(-\infty) \hat{P}_{1}^{*}(0)
\end{aligned}
$$

and if $\tau$ is large enough the dominant term of $\mathscr{P}_{21}$ as $\epsilon$ tends to zero will be given by

$$
\left\|\hat{P}_{2}^{*}(0) \hat{A}_{*}(\infty) \hat{A}_{*}^{-1}(-\infty) \hat{P}_{1}^{*}(0)\right\|^{2}
$$

But this expression is the transition probability of the following two-dimensional problem in $Q_{*}(0) \mathscr{H}$ : Let $\psi(t)$ be a normalized solution of the equation

$$
i \epsilon \frac{\partial}{\partial t} \psi(t)=\hat{H}_{*}(t) \psi(t)
$$

such that

$$
\lim _{t \rightarrow-\infty}\left\|\hat{P}_{1}^{*}(t) \psi(t)\right\|=1
$$

Then, by a computation similar to the one above,

$$
\hat{\mathscr{P}}_{21} \equiv \lim _{t \rightarrow-\infty}\left\|\hat{P}_{2}^{*}(t) \psi(t)\right\|^{2}=\left\|\hat{P}_{2}^{*}(0) \hat{A}_{*}(\infty) \hat{A}_{*}^{-1}(-\infty) \hat{P}_{1}^{*}(0)\right\|^{2}
$$

is the transition probability from $\hat{P}_{1}^{*}(-\infty)$ to $\hat{P}_{1}^{*}(+\infty)$. We have therefore reduced the initial problem to an effective two-dimensional problem.

\section{B. Asymptotic formula for the transition probability $\mathscr{P}_{21}$ in the adiabatic limit}

In this paragraph we compute an asymptotic expression for the transition probability $\hat{\mathscr{P}}_{21}$ of the problem (3.34) and (3.35). If the decay rate $\tau$ is large enough, then $\mathscr{P}_{21}$ has the same asymptotic expression. This is what we mean by the condition stating that the two levels must be sufficiently isolated in the spectrum. We assume that conditions $\mathrm{V}$ and VI are also satisfied. We recall that $W_{*}(t)$ is defined by

$$
i \frac{\partial}{\partial t} W_{*}(t)=K_{N *}(t) W_{*}(t), \quad W_{*}(0)=\mathbf{I}
$$

and that 


$$
\hat{H}_{*}(t)=W_{*}^{-1}(t) H_{*}(t) W_{*}(t)
$$

with

$$
H_{*}(t)=H(t)-\epsilon K_{N *-1}(t)
$$

In this section we consider only the operator $\hat{H}_{*}(l)$ on the time-independent two-dimensional subspace $Q_{*}(0) \mathscr{H}$. If $\epsilon$ is small enough, then $\hat{H}_{*}(t)$ has two separated eigenvalues $e_{1}^{*}(t)$ and $e_{2}^{*}(t)$. By the perturbation theory and proposition 2.1

$$
e_{j}^{*}(t)=e_{j}(t)+O\left(\epsilon\left\|K_{N_{-1}}(t)\right\|\right)=e_{j}(t)+O(\epsilon b(t)), \quad j=1,2,
$$

with $b(t)$ the integrable function of condition II. The corresponding spectral projectors are

$$
\hat{P}_{j}^{*}(t)=W_{*}^{-1}(t) P_{j}^{*}(t) W_{*}(t), \quad j=1,2 .
$$

We analyze the boundary problem

$$
\begin{gathered}
i \epsilon \frac{\partial}{\partial t} \psi(t)=\hat{H}_{*}(t) \psi(t), \\
\lim _{t \rightarrow-\infty}\left\|\hat{P}_{1}^{*}(t) \psi(t)\right\|=1, \quad\|\psi(t)\|=1
\end{gathered}
$$

by the method developed in Ref. 1. We first show that Eqs. (3.42) can be studied in the band $\Omega$, provided $\epsilon$ is small enough.

Lemma 3.1: Let $0<\rho<r$ be given. Then, there exists $\epsilon(\rho)>0$ such that for all $\epsilon<\epsilon(\rho)$, $\hat{H}_{*}(t)$ has an analytic continuation in a neighborhood of $\{z=t+i s|| s \mid \leqslant \rho\}$.

Proof: It is sufficient to show that $K_{N^{*}-1}$ and $K_{N^{*}}$ have analytic continuations in a neighborhood of $\{z=t+i s|| s \mid \leqslant \rho\}$, since this implies that $H_{*}$ and $W_{*}$ have analytic continuations in the same neighborhood. For large values of $|t|$ the analytic continuation follows from proposition 2.1 , since we can apply it with $D(z, \eta)$ replaced by $\{z=t+i s|| s \mid \leqslant \rho, t>R\}$ or $\{z=t+i s|| s \mid \leqslant \rho, t<-R\}$, by choosing $R$ large enough. Indeed we can verify the hypothesis of this proposition using condition IV. Let us consider the compact set $\Omega=\{z=t$ $+i s|| s|<\rho| t \mid,<R\}$. For each $z \in \Omega$ we can find a $\eta(z)$ so that proposition 2.1 applies. Therefore the union of the open discs $D(z, \eta(z))$ is a covering of $\Omega$ and consequently we can cover $\Omega$ by a finite number of discs $D(z, \eta(z))$. Thus there exists a nonzero $\epsilon(\rho)$ so that, for all $\epsilon<\epsilon(\rho)$, $K_{N^{*}-1}$ and $K_{N^{*}}$ have analytic continuation in a neighborhood of $\{z=t+i s|| s \mid \leqslant \rho\}$.

In the band $\Omega$ there is an eigenvalue-crossing point for $e_{1}(z)$ and $e_{2}(z)$ at $z^{*}$ with $\operatorname{Im} z^{*}>0$. Let $0<r^{\prime}<r$ be given with $\operatorname{Im} z^{*}<r^{\prime}$. By Rouche's theorem there is exactly one eigenvalue crossing point $\hat{z}^{*}$ for $e_{1}^{*}(z)$ and $e_{2}^{*}(z)$ in the band $\left\{z=t+i s \mid 0 \leqslant s \leqslant r^{\prime}\right\}$, which is near $z^{*}$ provided $\epsilon$ is small enough. Moreover the singularity of $e_{j}^{*}(z)$ at $z^{*}$ is of the same type as the one of $e_{j}(z)$ at $z^{*}$. Let $\gamma$ be a closed path as in Fig. 4. Let $\psi_{j}\left(t_{0}\right), j=1,2$ be two normalized eigenvectors of $H\left(t_{0}\right)$ with eigenvalues $e_{j}\left(t_{0}\right)$. Let $\hat{\psi}_{j}^{*}\left(t_{0}\right)$, resp. $\psi_{j}^{*}\left(t_{0}\right), j=1,2$, be two normalized eigenvectors of $\hat{H}_{*}\left(t_{0}\right)$, resp. $H_{*}\left(t_{0}\right)$ with eigenvalues $e_{j}^{*}\left(t_{0}\right)$. The phases of $\hat{\psi}_{j}^{*}(t)$ are fixed as in Eq. (3.5). We make an analytic continuation of all these objects along the path $\gamma$ and we denote with $a \sim$ the result of the analytic continuation when we come back to $t_{0}$. We know that

$$
\widetilde{e}_{1}\left(t_{0}\right)=e_{2}\left(t_{0}\right)
$$

and

$$
\widetilde{\psi}_{1}\left(t_{0}\right)=\exp \left(-i \theta_{21}\left(t_{0} \mid \gamma\right)\right) \psi_{2}\left(t_{0}\right)
$$




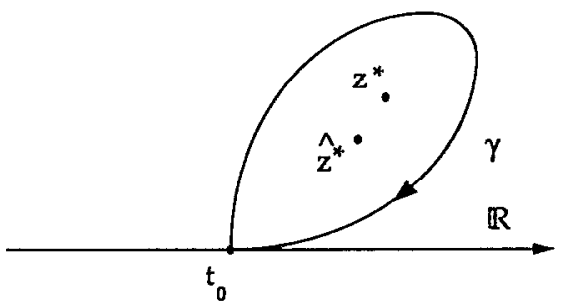

FIG. 4. The path $\gamma$ encircling $z^{*}$ and $z^{*}$.

For small enough $\epsilon$ we also have

$$
\tilde{e}_{1}^{*}\left(t_{0}\right)=e_{2}^{*}\left(t_{0}\right)
$$

and therefore

$$
\widetilde{\psi}_{1}^{*}\left(t_{0}\right)=\exp \left(-i \theta_{21}^{*}\left(t_{0} \mid \gamma\right)\right) \psi_{2}^{*}\left(t_{0}\right)
$$

and

$$
\widetilde{\hat{\psi}}_{1}^{*}\left(t_{0}\right)=\exp \left(-i \hat{\theta}_{21}^{*}\left(t_{0} \mid \gamma\right)\right) \hat{\psi}_{2}^{*}\left(t_{0}\right)
$$

where $\hat{\psi}_{j}^{*}=W_{*}^{-1} \psi_{j}^{*}$. Since $W_{*}(t)$ is analytic in the band we conclude that

$$
\theta_{21}^{*}\left(t_{0} \mid \gamma\right)=\hat{\theta}_{21}^{*}\left(t_{0} \mid \gamma\right)
$$

By perturbation theory again, we can write

$$
\psi_{j}^{*}\left(t_{0}\right)=\psi_{j}\left(t_{0}\right)+\chi_{j}\left(t_{0}\right), \quad j=1,2,
$$

with

$$
\left\|\chi_{j}\left(t_{0}\right)\right\|=O(\epsilon)
$$

Therefore we have

$$
\begin{aligned}
\widetilde{\psi}_{1}^{*}\left(t_{0}\right) & =\exp \left(-i \theta_{21}^{*}\left(t_{0} \mid \gamma\right)\right) \psi_{2}^{*}\left(t_{0}\right) \\
& =\exp \left(-i \theta_{21}^{*}\left(t_{0} \mid \gamma\right)\right)\left(\psi_{2}\left(t_{0}\right)+\chi_{2}\left(t_{0}\right)\right) \\
& =\widetilde{\psi}_{1}\left(t_{0}\right)+\widetilde{\chi}_{1}\left(t_{0}\right) \\
& =\exp \left(-i \theta_{21}\left(t_{0} \mid \gamma\right)\right) \psi_{2}\left(t_{0}\right)+\widetilde{\chi}_{1}\left(t_{0}\right)
\end{aligned}
$$

After analytic continuation we still have $\left\|\tilde{\chi}_{1}\left(t_{0}\right)\right\|=O(\epsilon)$. Therefore we conclude that

$$
\exp \left(i \theta_{21}^{*}\left(t_{0} \mid \gamma\right)-i \theta_{21}\left(t_{0} \mid \gamma\right)\right)=1+O(\epsilon)
$$

and thus

$$
\theta_{21}^{*}\left(t_{0} \mid \gamma\right)=\theta_{21}\left(t_{0} \mid \gamma\right)+O(\epsilon)
$$


It remains to show that the generalized Dykhne formula is valid for our problem (3.42). According to Ref. 1 it is sufficient to show the existence of a path $t \in \mathbb{R} \mapsto \eta(t)$ in $\Omega$, such that

(i) $\lim _{t \rightarrow \pm \infty} \operatorname{Re} \eta(t)= \pm \infty, \lim _{\mathrm{t} \rightarrow \pm \infty} \operatorname{Im} \eta(t)=s_{ \pm}$with $s_{ \pm}<r$,

(ii) $\eta$ goes above the eigenvalue-crossing $\hat{z}^{*}$,

(iii) along the path $\eta(t) \operatorname{Im} \Delta_{12}^{*}(\eta(t))$ is a nondecreasing function.

The function $\Delta_{12}^{*}(z)$ is defined as the analytic continuation of the function

$$
\int_{t_{0}}^{z}\left(e_{1}^{*}\left(z^{\prime}\right)-e_{2}^{*}\left(z^{\prime}\right)\right) d z^{\prime}
$$

Condition VI implies (see Ref. 1) the existence of such a path $\zeta$ when we replace $\hat{z}^{*}$ by $z^{*}$ and $\Delta_{21}^{*}$ by $\Delta_{21}$ which is given by

$$
\int_{t_{0}}^{z}\left(e_{1}\left(z^{\prime}\right)-e_{2}\left(z^{\prime}\right)\right) d z^{\prime}
$$

Let $\zeta$ be such a path and $\rho$ be large enough so that

$$
|\operatorname{Im} \zeta(t)|<\rho, \quad t \in \mathbb{R} .
$$

Using a perturbative argument, as in Ref. 2 , we can prove the existence of a path $\eta$ with

$$
|\operatorname{Im} \eta(t)|<\rho, \quad t \in \mathbb{R}
$$

satisfying the above properties. It is then straightforward to check that the integration by parts procedure used in Ref. 1 gives uniform bounds in $\epsilon$ when performed on $\hat{H}_{*}$. Therefore, there exists some $\epsilon^{*}$, such that for all $\epsilon<\epsilon^{*}$

$$
\hat{\mathscr{P}}_{21}(\epsilon)=\exp \left(2 \operatorname{Im} \hat{\theta}_{21}^{*}\right) \exp \left(2 \epsilon^{-1} \operatorname{Im} \int_{\gamma} e_{1}^{*}\right)(1+O(\epsilon))
$$

Note that $e_{1}^{*}(t)$ is the eigenvalue of the operator $H_{*}(t)$ which can be written as

$$
H_{*}(t)=H(t)-\epsilon K_{0}(t)+\epsilon\left(K_{0}(t)-K_{N_{-1}}(t)\right)=H(t)-\epsilon K_{0}(t)+O\left(\epsilon^{2}\right)
$$

Let $\psi_{1}(t)$ be the eigenvector of $H(t)$ for the eigenvalue $e_{1}(t)$. Then

$$
e_{1}^{*}(t)=e_{1}(t)-\epsilon\left\langle\psi_{1}(t) \mid K_{0}(t) \psi_{1}(t)\right\rangle+O\left(\epsilon^{2}\right)
$$

The term of first order in $\epsilon$ vanishes because

$$
P_{1}(t)\left[Q^{\prime}(t), Q(t)\right] P_{1}(t)=0
$$

since for any projector $P(t)$ we have

$$
P(t) P^{\prime}(t) P(t)=0 .
$$

Therefore we have 


$$
\hat{P}_{21}(\epsilon)=\exp \left(2 \operatorname{Im} \theta_{21}\right) \exp \left(2 \epsilon^{-1} \operatorname{Im} \int_{\gamma} e_{1}\right)(1+O(\epsilon))
$$

provided $\epsilon$ is small enough.

\section{CONCLUSION}

Let us briefly summarize the results and methods we used in this study of the adiabatic regime.

We first consider the construction of a superadiabatic approximation when the Hamiltonian possesses a gap in its spectrum by means of a local divergent iterative scheme. We make use of regular perturbation theory to control its terms in an efficient way and obtain their behavior as a function of both the parameter $\epsilon$ and their index $n$. The superadiabatic evolution is then obtained by an optimal truncation at fixed $\epsilon$ of the iterative scheme. An important point of this construction is that we get useful estimates on the accuracy of the superadiabatic approximation as a function of the gap. As a by-product we obtain the physically expected behavior of the exponentially small transition probability across the gap, as a function of gap: the exponential decay rate is proportional to the width of the gap (see the remarks below theorem 2.1).

The second part of the paper is a combination of the above technique and previous techniques to compute the leading term of the transition probability between two nondegenerate levels $e_{1}(t)$ and $e_{2}(t)$ well separated from the rest of the spectrum. As a first step, we use our superadiabatic evolution to decouple the transitions between the two levels from the transitions out of the two-dimensional eigenspace $Q(t) \mathscr{H}$ they live in: we reduce the computation of the initial transition probability to the computation of the transition probability for an effective two-level system, up to corrections which are shown to be negligible if the two levels are sufficiently isolated in the spectrum. This last property which is expected on physical grounds comes from the estimates as functions of the gap in the first part of the paper. As a second step, we apply the results of Ref. 1 to the effective two-level problem. The end result is an explicit formula for the transition probability between $e_{1}$ and $e_{2}$ given by a decreasing exponential $\exp (-\gamma / \epsilon)$, with $\gamma$ depending on the energy levels $e_{j}(t)$ only, multiplied by a geometric prefactor. We also give an explicit formula for the geometrical prefactor in terms of the matrix elements of the initial Hamiltonian in the two-dimensional eigenspace $Q(t) \mathscr{H}$. An important feature of the expression yielding the geometric prefactor is that it depends on the embedding in the total Hilbert space of the two-dimensional space spanned by the eigenvectors of energies $e_{1}(t)$ and $e_{2}(t)$.

\section{ACKNOWLEDGMENTS}

We have benefited from an earlier correspondence with $M$. Berry about the asymptotic properties of our iterative scheme and from several discussions with G. Nenciu about his latest results. We thank M. Berry and G. Nenciu very much for these fruitful exchanges.

This research was supported by Fonds National Suisse de la Recherche, Grant No. 2028521.90 .

\section{APPENDIX}

We derive in this appendix the explicit formula for $\operatorname{Im} \theta_{21}$ given in proposition 3.1 . We start by considering the existence of a suitable basis of $Q(t) \mathscr{H}$.

Let $\eta_{1}$ and $\eta_{2}$ belong to the range of $Q(0)$. We define for $t \in \mathbb{R}$

$$
\varphi_{1}(t)=\frac{Q(t) \eta_{1}}{\sqrt{\left\langle\eta_{1} \mid Q(t) \eta_{1}\right\rangle}},
$$




$$
\varphi_{2}(t)=\frac{Q(t)\left(\eta_{2}-\left(\left\langle\eta_{1} \mid Q(t) \eta_{2}\right\rangle /\left\langle\eta_{1} \mid Q(t) \eta_{1}\right\rangle\right) \eta_{1}\right)}{\sqrt{\left\langle\eta_{2} \mid Q(t)\left(\eta_{2}-\left(\left\langle\eta_{1} \mid Q(t) \eta_{2}\right\rangle /\left\langle\eta_{1} \mid Q(t) \eta_{1}\right\rangle\right) \eta_{1}\right)\right\rangle}}
$$

These vectors form an orthonormal basis of $Q(t) \mathscr{H}$ for $t$ close to 0 and they are analytic in $t$ by assumptions $I$ and $V$. It should be noticed that the basis $\left\{\varphi_{1}(t), \varphi_{2}(t)\right\}$ is time dependent because $Q(t)$ is. Hence we have the representation (3.10) of $H(t)$ in the subspace $Q(t) \mathscr{K}$ with the definitions (3.11). The expressions

$$
\frac{\left\langle\eta_{1} \mid H(t) Q(t) \eta_{1}\right\rangle}{\left\langle\eta_{1} \mid Q(t) \eta_{1}\right\rangle}
$$

and

$$
\left\langle\varphi_{1}(t) \mid H(t) \varphi_{2}(t)\right\rangle=\frac{\left\langle\eta_{1} \mid H(t) Q(t) \varphi_{2}(t)\right\rangle}{\sqrt{\left\langle\eta_{1} \mid Q(t) \eta_{1}\right\rangle}}
$$

have analytic extensions in the complex plane, so that the same is true for their real or imaginary parts considered as real analytic functions on the real axis. Thus the magnetic field $\mathbf{B}(z)$ is analytic in $z \in \Omega$. We introduce the vectors

$$
\begin{aligned}
\chi_{j}(t) & =\left(B_{3}(t)+(-1)^{j} \sqrt{\rho(t)}\right) \varphi_{1}(t)+\left(B_{1}(t)+i B_{2}(t)\right) \varphi_{2}(t) \\
& \equiv \alpha_{j}(t) \varphi_{1}(t)+\beta(t) \varphi_{2}(t), j=1,2
\end{aligned}
$$

which satisfy by construction

$$
H(t) \chi_{j}(t)=e_{j}(t) \chi_{j}(t), \quad j=1,2
$$

Moreover, their analytic continuations along $\sigma$, from the origin back to the origin, are given by

$$
\bar{\chi}_{j}(0)=\chi_{k}(0)
$$

since the components $B_{k}(t)$ and the basis vectors $\varphi_{j}(t)$ have analytic continuations in a neighborhood of the origin, even at $z=z^{*}$. As in Ref. 1 we write the eigenvector $\psi_{j}(t)$ satisfying

$$
\left\langle\psi_{j}(t) \mid \psi_{j}^{\prime}(t)\right\rangle \equiv 0
$$

under the form

$$
\psi_{j}(t) \equiv \exp \left\{-i \delta_{j}(t)\right\} \chi_{j}(t), \quad j=1,2,
$$

with $\exp \left\{i \delta_{j}(0)\right\}=\left\|\chi_{j}(0)\right\|$. It then follows from Eq. (A6) that

$$
\operatorname{Im} \theta_{21}=\operatorname{Im}\left(\int_{\sigma} \delta_{1}^{\prime}(z)+\delta_{1}(0)-\delta_{2}(0)\right)
$$

We have by condition (A7)

$$
i \delta_{j}^{\prime}(t)=\frac{\left\langle\chi_{j}(t) \mid \chi_{j}^{\prime}(t)\right\rangle}{\left\langle\chi_{j}(t) \mid \chi_{j}(t)\right\rangle} \forall t \in \mathbb{R}
$$


Due to the time dependence of the basis vectors $\varphi_{j}(t)$, we obtain for $\delta_{j}^{\prime}$ (without expliciting the argument $t, t \in \mathbb{R}$ )

$$
\begin{aligned}
i \delta_{j}^{\prime}= & \frac{1}{\left|\alpha_{j}\right|^{2}+|\beta|^{2}}\left(\bar{\alpha}, \alpha_{j}^{\prime}+\bar{\beta} \beta^{\prime}\right)+\frac{1}{\left|\alpha_{j}\right|^{2}+|\beta|^{2}}\left\{\left|\alpha_{j}\right|^{2}\left\langle\varphi_{1} \mid \varphi_{1}^{\prime}\right\rangle+|\beta|^{2}\left\langle\varphi_{2} \mid \varphi_{2}^{\prime}\right\rangle+\bar{\alpha} \beta\left\langle\varphi_{1} \mid \varphi_{2}^{\prime}\right\rangle\right. \\
& \left.+\bar{\beta} \alpha_{j}\left\langle\varphi_{2} \mid \varphi_{1}^{\prime}\right\rangle\right\} .
\end{aligned}
$$

The first term coincides with the previously computed one in Ref. 1. Using the self-adjointness of $Q(t)$ for $t \in \mathbb{R}$, we check that the functions

$$
\left\langle\varphi_{j}(t) \mid \varphi_{k}^{\prime}(t)\right\rangle \quad t \in \mathbb{R}
$$

have analytic continuations close to the origin, even at $z=z^{*}$, which we denote by

$$
\left\langle\varphi_{j} \mid \varphi_{k}^{\prime}\right\rangle(z) \text {. }
$$

Multiplying Eq. (A11) by $\left(\sqrt{\rho}+B_{3}\right) /\left(\sqrt{\rho}+B_{3}\right)$, using the result of the appendix in Ref. 1 and Eq. (A9) we obtain

$$
\begin{aligned}
\operatorname{Im} \theta_{21}= & \operatorname{Im} \int_{\sigma} \frac{B_{3}\left(B_{1} B_{2}^{\prime}-B_{2} B_{1}^{\prime}\right)}{2 \sqrt{\rho}\left(B_{1}^{2}+B_{2}^{2}\right)} d z+\operatorname{Re} \int_{\sigma}\left(\frac{\left(B_{3}-\sqrt{\rho}\right)\left(B_{1}^{2}+B_{2}^{2}\right)}{2 \sqrt{\rho}\left(B_{1}^{2}+B_{2}^{2}\right)}\left\langle\varphi_{1} \mid \varphi_{1}^{\prime}\right\rangle\right. \\
& -\frac{\left(B_{3}+\sqrt{\rho}\right)\left(B_{1}^{2}+B_{2}^{2}\right)}{2 \sqrt{\rho}\left(B_{1}^{2}+B_{2}^{2}\right)}\left\langle\varphi_{2} \mid \varphi_{2}^{\prime}\right\rangle+\frac{\left(B_{1}^{2}+B_{2}^{2}\right)\left(B_{1}+i B_{2}\right)}{2 \sqrt{\rho}\left(B_{1}^{2}+B_{2}^{2}\right)}\left\langle\varphi_{1} \mid \varphi_{2}^{\prime}\right\rangle \\
& \left.+\frac{\left(B_{1}^{2}+B_{2}^{2}\right)\left(B_{1}-i B_{2}\right)}{2 \sqrt{\rho}\left(B_{1}^{2}+B_{2}^{2}\right)}\left\langle\varphi_{2} \mid \varphi_{1}^{\prime}\right\rangle\right) d z
\end{aligned}
$$

for a loop $\sigma$ containing no zero of $\left(B_{1}^{2}+B_{2}^{2}\right)$. This proves the proposition.

Let us finally consider the vectors

$$
\Phi_{j}(t) \equiv W(t) \eta_{j}, \quad j=1,2
$$

where $W(t)$ is defined by

$$
i W^{\prime}(t)=i\left[Q^{\prime}(t), Q(t)\right] W(t), \quad W(0)=\mathbb{I}
$$

and $\eta_{j}$ form an orthonormal basis of $Q(0) \mathscr{H}$. Since $W(z) Q(0)=Q(z) W(z)$ [see Eq. (3.18)] and $Q(z)$ is analytic in $\Omega$, these vectors form an orthonormal basis of $Q(t) \mathscr{H}, t \in \mathbb{R}$, which is analytic in $\Omega$. We compute for real $t$

$$
\begin{aligned}
\left\langle\Phi_{j}(t) \mid \Phi_{k}^{\prime}(t)\right\rangle & =\left\langle\Phi_{j}(t) \mid\left[Q^{\prime}(t), Q(t)\right] \Phi_{k}(t)\right\rangle \\
& =\left\langle\Phi_{j}(t) \mid Q(t)\left[Q^{\prime}(t), Q(t)\right] Q(t) \Phi_{k}(t)\right\rangle \\
& \equiv 0 \quad \forall j, k=1,2 .
\end{aligned}
$$

Hence the factor $\operatorname{Im} \theta_{21}$ has the same expression in terms of the matrix elements of $H(t)$ in the basis $\left\{\Phi_{1}(t), \Phi_{2}(t)\right\}$, as in the two-level case. 
${ }^{1}$ A. Joye, H. Kunz, and C.-E. Pfister, Ann. Phys. 208, 299 (1991).

${ }^{2}$ A. Joye, and C.-E. Pfister, J. Phys. A 24, 753 (1991).

${ }^{3}$ A. Joye, G. Mileti, and C.-E. Pfister, Phys. Rev. A 44, 4280 (1991).

${ }^{4}$ A. Joye and C.-E. Pfister, Commun. Math. Phys. 140, 15 (1991).

${ }^{5} \mathrm{G}$. Nenciu, Linear Adiabatic theory and applications: Exponential Estimates, Preprint (1991).

${ }^{6}$ L. M. Garrido, J. Math. Phys. 6, 335 (1964).

${ }^{7}$ M. V. Berry, Proc. R. Soc London, Ser. A 414, 31 (1987).

M. V. Berry, Proc. R. Soc London, Ser. A 429, 61 (1990).

${ }^{9}$ M. V. Berry, Proc. R. Soc. London, Ser. A 430, 405 (1990).

${ }^{10}$ J. W. Zwanziger, S. P. Rucker, and G. C. Chingas, Phys. Rev. A 43, 3232 (1991).

${ }^{11} \mathrm{~S}$. G. Krein, Translations of Mathematical Monographs (American Mathematical Society, Providence, 1971), Vol. 29. 